\title{
Evaluation of the concordance in HPV type between self- and physician-collected samples using a brush-based device and a PCR-based HPV DNA test in Japanese referred patients with abnormal cytology or HPV infection
}

\author{
Toshimichi Onuma $^{1}\left[\right.$ - Tetsuji Kurokawa ${ }^{1} \cdot$ Akiko Shinagawa $^{1} \cdot$ Yoko Chino $^{1} \cdot$ Yoshio Yoshida $^{1}$
}

Received: 17 December 2019 / Accepted: 11 June 2020 / Published online: 24 June 2020

(c) The Author(s) 2020

\begin{abstract}
Background To adopt HPV self-sampling in Japan, we assessed the concordance between self- and physician-collected human papillomavirus (HPV) samples from Japanese patients and examined the performance of HPV self-sampling for cervical intraepithelial neoplasia grade 2 or worse (CIN2+).

Methods Patients who had previously tested negative for intraepithelial lesions or malignancy/HPV-positive, and patients with atypical squamous cells of undetermined significance or worse (ASCUS+) cytology were eligible for this cross-sectional study. Participants performed HPV self-sampling using an Evalyn brush, which was submitted at the Fukui Prefectural Health Care Association. The Evalyn brush heads were stored in ThinPrep vials. The physician, however, performed HPV and cell sampling using an endocervical brush and immediately stored the brush heads in ThinPrep vials. All participants underwent colposcopy and biopsy. Histopathological diagnoses were made by pathologists at Fukui University Hospital. HPV infection was confirmed using a PCR-based Cobas 4800 HPV DNA test. Cytological analysis was performed at Fukui Prefectural Health Care Association.

Results HPV-positive rates for physician-collected samples and self-collected samples were 51 and $50 \%$, respectively. The perfect match rate of HPV type between the groups was $88 \%(\kappa=0.76)$. HPV16/18 showed higher agreement rates than other HPVs (99\%, kappa 0.96 and 89\% kappa 0.77, respectively). Both groups showed $100 \%$ sensitivity to CIN2+, but specificity was 57.0 and $58.1 \%$, respectively.

Conclusion For HPV typing, a good concordance rate was seen between self- and physician-collected samples. Self-sampling showed high sensitivity for CIN2+. Self-sampling using the Evalyn brush and Cobas 4800 may be feasible for screening Japanese individuals.
\end{abstract}

Keywords Cervical cancer $\cdot$ Screening $\cdot$ HPV self-sampling $\cdot$ Brush $\cdot$ PCR-based HPV test

\section{Introduction}

Japan has a higher incidence of age-adjusted cervical cancer than the United States and Australia, where screenings are organized [1]. The mortality rate of cervical cancer patients aged $<50$ years is increasing, with the high incidence attributable to the low human papillomavirus (HPV) vaccination

Toshimichi Onuma

toonuma@u-fukui.ac.jp

1 Department of Obstetrics and Gynecology, Faculty of Medical Sciences, University of Fukui, 23-3 Matsuoka-Shimoaizuki, Eiheiji-cho, Fukui 910-1193, Japan rate and low screening rate in Japan [2]. Most cervical cancers occur in unscreened women [3]; therefore, it is necessary to increase the number of women screened for cervical cancer to reduce its incidence in Japan.

Epidemiological studies using molecular technology show that persistent infection with high-risk HPV (hrHPV) is strongly associated with development of cervical intraepithelial neoplasia (CIN) and cervical cancer [4]. Therefore, HPV testing has been introduced alone or combined with cytology for cervical cancer screening. Physician-collected hrHPV tests are more sensitive for detecting CIN2 or worse $(\mathrm{CIN} 2+)$ than cytology alone [5]. Women who were physician-collected HPV - at baseline have lower rates of 
CIN2+ at 48 months relative to cytology negative women at baseline [6]. Primary physician-collected HPV screening every 5 years with partial genotyping is predicted to be substantially more effective and potentially cost-saving relative to current cytology screening programs undertaken every 2 years [7]. The Netherlands and Turkey fully implemented national HPV-based cervical cancer screening [8].

HPV self-sampling is a screening method, where HPV sampling is performed by the screening participant and displays high HPV test concordance with HPV physician sampling in various referral and screening populations [9]. HPV self-sampling using polymerase chain reaction (PCR)based HPV testing is almost equivalent to HPV physician sampling in sensitivity to CIN2+ and displays greater sensitivity for CIN2+ relative to physician-collected cytology [10]. Additionally, HPV self-sampling is less awkward than physician screening and acceptable for screening patients [11]. A previous study of HPV self-sampling for unscreened women showed that self-sampling significantly improved participation in the screening program $[12,13]$. Therefore, HPV self-sampling was adopted as part of the cervical cancer screening program in the Netherlands [14].

Several HPV self-sampling devices and detection methods are available [15]. HPV self-sampling with brush- and lavage-based self-collection devices show increased sensitivity for CIN2+ relative to sampling performed with swab- or tampon-based self-collection [10]. Among self-sampling brush devices, the Evalyn brush shows high performance and good patient acceptance [16, 17]. A recent meta-analysis reported that the hybrid capture (HC) method is less sensitive for CIN2+ than the HPV DNA PCR method for HPV self-sampling [15]. To apply self-sampling in clinical practice, it is important to use clinically evaluated PCR-based HPV testing. The Cobas 4800 is a clinically established PCR-based HPV test [18] that shows good HPV test performance for CIN detection in referral populations [19, 20]. However, the HPV concordance rate and CIN2+-detection sensitivity using the Evalyn brush and Cobas 4800 in the Japanese population have not been reported. Furthermore, the study population using these methods involved patients with premalignant lesions, cervical carcinoma, and carcinoma suspicion [19] or patients with atypical squamous cells of undetermined significance (ASCUS) [20]. Additionally, CIN has been detected in negative for intraepithelial lesions or malignancy (NILM)/HPV-positive Japanese patients [21]. Introduction of HPV self-sampling for screening requires confirmation of consistency with results of physician sampling of NILM/HPV-positive patients. However, there are no reports using the Evalyn brush and/or the Cobas 4800 showing concordance rates between self-collection and physician collection in NILM/HPV-positive groups.

We hypothesized that HPV self-sampling with the Evalyn brush and Cobas 4800 PCR-based HPV testing would show good HPV-type agreement between physician sampling and self-sampling along with high detection sensitivity of CIN2+ in the Japanese population. To test this hypothesis, we compared HPV self-sampling using the Evalyn brush and Cobas 4800 with physician sampling performed in a Japanese referral population that included NILM/HPV patients.

\section{Patients and methods}

\section{Study population}

We conducted a cross-sectional study comparing the results of HPV self-sampling with physician sampling at the University of Fukui Hospital from January 2019 to July 2019. Previous studies report that referral populations sampled by physicians showed an hrHPV-infection rate of $\sim 20$ to $50 \%$, resulting in colposcopy, regardless of cytology results [9, 15]. To obtain a referral population with the same HPVinfection rate as previous studies, as well as NILM/HPV patients, we included two patient types: (1) outpatients with abnormal cytology and requiring colposcopy and biopsy and (2) NILM/HPV-positive patients in the Fukui Cervical Cancer Study (FCCS). The FCCS investigated whether combined screening with liquid-based cytology (LBC) and HPV testing could be useful in Japan within the framework of actual screening. After an initial visit, NILM/HPV-positive patients in the baseline phase of the FCCS study were followed up for 3 years and consistently underwent physician-collected HPV testing, cytology, and colposcopy at the annual visit, unless they showed CIN3 or worse (CIN3+) [21]. Exclusion criteria included patients who had undergone hysterectomy, were pregnant, or who had received chemotherapy. Patients not excluded provided written informed consent for participation following explanation of the study design. This study was approved by the Fukui University Hospital Ethics Committee (no. 20180080).

\section{Sample collection}

Participants received instruction on how to submit samples after HPV self-sampling but not details concerning use of the Evalyn brush for sample collection. Participants were instructed to read the instructions describing use of the Evalyn brush before self-sampling, with these instructions created under supervision of the Japan Cancer Society. These instructions were verified that Japanese people could read and understand before this study. Participants performed HPV self-sampling in the bathroom at the hospital and then submitted the brushes. Immediately after HPV self-sampling, HPV physician sampling and cytology were performed. The Rovers Cervex brush (Rovers Medical Devices, Oss, The Netherlands) was used for physician 
sampling. After sampling, the Cervex brush was immediately placed in ThinPrep vials (Hologic, Marlborough, MA, USA), and colposcopy and biopsy were performed. All physicians involved in sampling were gynecologic oncology specialists. The Evalyn brush and physician-sampled ThinPrep vials were stored at room temperature and transferred to the Fukui Health Care Association on a fixed day of the week. Cytologists examined the cytology samples using the LBC method at the Fukui Health Care Association. Two pathologists at Fukui University Hospital examined the colposcopic biopsy tissue. Cytologists and pathologists were not informed of HPV test results prior to diagnosis.

\section{HPV testing}

The Evalyn brush was processed after transfer to the Fukui Health Care Association. The Evalyn brush was placed into ThinPrep vials and stirred to release the cells. Both physician- and self-sampled ThinPrep vials were stored at room temperature until measurement. HPV testing was performed using the Cobas 4800 system. HPV-16 and -18 as well as 12 other HPV genotypes, including $-31,-33,-35,-39,-45$, $-51,-52,-56,-58,-59,-66$, and -68 , were measured. Previously reported methods were used for all measurements $[18,21]$. HPV testing was performed without information on patient background, cytology results, or histology results.

\section{Statistical analysis}

Continuous variables are presented as the mean \pm standard deviation, and categorical variables are expressed as frequencies and proportions. Student's $t$ test was used to compare continuous variables. Agreement rates of perfect matches for HPV typing between the self- and physiciancollected samples were examined, as were agreement rates for HPV-16/-18 and HPV others. HPV concordance between paired samples was assessed using the Kappa statistic (Cohen's Kappa; $\kappa$ ) and defined as "Poor" $(\kappa \leq 0.20)$, "Fair" $(0.21 \leq \kappa \leq 0.40)$, "Moderate" $(0.41 \leq \kappa \leq 0.60)$, "Good" $(0.61 \leq \kappa \leq 0.80)$, or "Very good" $(\kappa \geq 0.81)$. The sensitivity and specificity of HPV self-sampling were calculated using HPV physician sampling as the standard [20]. CIN2+ was defined as CIN2, CIN3, adenocarcinoma (ADC) in situ (AIS), squamous cell carcinoma (SCC), or ADC. CIN2+-detection sensitivity, specificity, positive predictive value (PPV), and negative predictive value (NPV) were calculated for both sample sets. ASCUS+ was defined as ASCUS, low-grade squamous intraepithelial lesion (LSIL), high-grade squamous intraepithelial lesion (HSIL), atypical squamous cells, cannot exclude HSIL (ASCH), SCC, atypical glandular cells (AGCs), and ADC. LSIL+ was defined as LSIL, ASCH, HSIL, SCC, AGC, or ADC. The detection sensitivity, specificity, PPV, and NPV for CIN2+ were calculated for ASCUS+ and LSIL+ patients. Data were analyzed using SPSS (v.21.0; IBM Corp., Armonk, NY, USA), and a $p<0.05$ was considered significant.

\section{Results}

\section{Patient characteristics}

Table 1 shows the age, cytological results, and histological results of participants. Patients aged 30-39 years were the majority (39\%: 39/100), followed by women aged $40-49$ years $(28 \%$; $28 / 100), 20-29$ years $(13 \% ; 13 / 100)$, and $50-59$ years $(13 \% ; 13 / 100)$. Two cases produced unsatisfactory Pap results. Cytological results were NILM $72.4 \%$ (71/98), ASCUS + 27.6\% (27/98), and LSIL+ $21.4 \%$ (21/98). One case did not undergo biopsy without colposcopic lesions. There were $73.7 \%$ (73/99) cases without dysplasia, $13.1 \%$ (13/99) of CIN1, 13.1\% (13/99) of CIN2+, and 7.1\% (7/99) of CIN3+.

Table 1 Patient characteristics

\begin{tabular}{ll}
\hline$n$ & 100 \\
Average age (SD) & $41.8(11.0)$ \\
Cytology & 71 \\
NILM & 6 \\
ASCUS & 6 \\
LSIL & 2 \\
ASCH & 9 \\
HSIL & 2 \\
SCC & 1 \\
AGC & 1 \\
ADC & 2 \\
Unsatisfactory Pap test & \\
Pathology & 73 \\
No dysplasia & 13 \\
CIN1 & 6 \\
CIN2 & 3 \\
CIN3 & 1 \\
SCC & 2 \\
AIS & 1 \\
ADC & 1 \\
No biopsy & \\
\hline
\end{tabular}

$A D C$ adenocarcinoma, $A G C$ atypical glandular cells, $A I S$ adenoma in situ, $A S C$ - $H$ atypical squamous cells, cannot exclude HSIL, ASCUS atypical squamous cells of undetermined significance, $C I N$ cervical intraepithelial neoplasia, HSIL high-grade squamous intraepithelial lesion, LSIL low-grade squamous intraepithelial lesion, NILM negative for intraepithelial lesion or malignancy, $S C C$ squamous cell carcinoma, $S D$ standard deviation 


\section{HPV type according to self- and physician sampling}

Table 2 shows the results of HPV type according to HPV self- and physician sampling. All self- and physician samples were valid for HPV testing. The HPV+ rates for selfand physician sampling were 50.0 and $51.0 \%$, respectively, with similar rates of different HPV types between sampling methods. Cases with multiple HPV infections were $4.0 \%$ (2/50) for self-sampling and 5.9\% (3/51) for physician sampling, with no cases with HPV-16 or -18 infections found. There were $11 \mathrm{HPV}+$ or HPV - discrepancies and one case of HPV-type discrepancy.

\section{Performance of HPV self-sampling relative to physician sampling}

Table 3 shows the performance of HPV self-sampling relative to physician sampling used as a reference standard. Coincidence was defined as a case when HPV type was the same. The HPV all type showed an agreement rate of $88 \%$ ( $\kappa$ : 0.76 ), sensitivity of $86 \%$ [95\% confidence interval (CI): 74-94\%], and specificity of 90\% (95\% CI 78-97\%). HPV16/-18 showed a higher agreement rate than HPV others $(99.0 \%, \kappa: 0.96$; and $89.0 \%, \kappa: 0.77$, respectively). Additionally, HPV-16/-18 showed higher sensitivity and specificity than HPV others (92.9 and 100\%; and 85.4 and $91.5 \%$, respectively).

\section{Discordant cases between self- and physician sampling}

Table 4 shows cases with inconsistent results for HPV testing between sampling methods. Overall, 91.7\% (11/12) of discordant cases were HPV others or HPV-, and $91.7 \%$ (11/12) were NILM. There were no cases with dysplasia among discordant cases, and the average age between concordant and discordant cases did not differ significantly $(42.1 \pm 11.1$ and $39.6 \pm 11.1$ years, respectively; $p=0.461)$. The number of days from self-sampling to HPV testing in all cases was $12.7 \pm 6.9$ days for HPV test-matched cases and $14.2 \pm 7.3$ days for HPV test-unmatched cases, although the difference was not significant $(p=0.482)$.

\section{CIN2+ detection sensitivities of self-sampling, physician sampling, and cytology}

Table 5 shows the CIN2+-detection sensitivity of HPV selfsampling, physician sampling, ASCUS+, and LSIL+. Selfsampling showed the same CIN2+-detection sensitivity and specificity as physician sampling (100 and 58.1\%; and 100 and $57.0 \%$, respectively). Self-sampling showed the same CIN2+-detection sensitivity but less specificity relative to cytology (self-sampling: 100 and 58.1\%; ASCUS+: 100 and 84.5\%; and LSIL+: 92.3 and 89.3\%). The coincidence rates between self-sampling and physician sampling for CIN2+
Table 2 HPV type in self- and physician sampling
Table 3 Performance of HPV self-sampling relative to physician sampling

\begin{tabular}{lclllll}
\hline & \multicolumn{2}{l}{$\mathrm{pHPV}$} & & & \\
\cline { 2 - 6 } & HPV (-) & HPV16 & HPV18 & HPV others & $\begin{array}{l}\text { HPV-16, } \\
\text { others }\end{array}$ & $\begin{array}{l}\text { HPV-18, } \\
\text { others }\end{array}$ \\
\hline sHPV & & & & & & \\
HPV (-) & 44 & 0 & 0 & 6 & 0 & 0 \\
HPV-16 & 0 & 4 & 0 & 0 & 0 & 0 \\
HPV-18 & 0 & 0 & 7 & 0 & 0 & 0 \\
HPV others & 5 & 0 & 0 & 31 & 1 & 0 \\
HPV-16, others & 0 & 0 & 0 & 0 & 1 & 0 \\
HPV-18, others & 0 & 0 & 0 & 0 & 0 & 1 \\
\hline
\end{tabular}

There were no cases of HPV-16 or -18 infection. HPV others includes $-31,-33,-35,-39,-45,-51,-52,-56$, $-58,-59$, and -66

$H P V$ human papillomavirus, $p H P V$ HPV physician sampling, sHPV HPV self-sampling

\begin{tabular}{llllc}
\hline & $\begin{array}{l}\text { Agreement } \\
\text { rate }(\%)\end{array}$ & Kappa (95\% CI) & Sensitivity (95\% CI) & Specificity (95\% CI) \\
\hline HPV, all types & 88.0 & $0.76(0.63-0.89)$ & $86.3(73.7-94.3)$ & $89.8(77.8-96.6)$ \\
HPV-6/18 & 99.0 & $0.96(0.87-1.0)$ & $92.9(66.1-99.8)$ & $100(93.8-100)$ \\
HPV others & 89.0 & $0.77(0.65-0.90)$ & $85.4(70.8-94.4)$ & $91.5(81.3-97.2)$ \\
\hline
\end{tabular}

HPV others includes $-31,-33,-35,-39,-45,-51,-52,-56,-58,-59$, and -66

CI, confidence interval; HPV, human papillomavirus; pHPV, HPV physician sampling; sHPV, HPV selfsampling 
Table 4 Discordant cases between self- and physician sampling

\begin{tabular}{lllll}
\hline Age (years) & sHPV & pHPV & Cytology & Pathology \\
\hline 29 & $(-)$ & Others & NILM & No dysplasia \\
29 & $(-)$ & Others & NILM & No dysplasia \\
31 & $(-)$ & Others & NILM & No dysplasia \\
35 & Others & $(-)$ & NILM & No dysplasia \\
35 & Others & $(-)$ & NILM & No dysplasia \\
36 & $(-)$ & Others & NILM & No dysplasia \\
36 & Others & Others, HPV-16 & HSIL & No dysplasia \\
38 & Others & $(-)$ & NILM & No dysplasia \\
42 & $(-)$ & Others & NILM & No dysplasia \\
44 & Others & $(-)$ & NILM & No dysplasia \\
52 & $(-)$ & Others & NILM & No dysplasia \\
68 & Others & $(-)$ & NILM & No dysplasia \\
\hline
\end{tabular}

Others includes HPV-31, -33, -35, -39, -45, -51, -52, -56, -58, -59, and -66

$H S I L$ high-grade squamous intraepithelial lesion, HPV human papillomavirus, NILM negative for intraepithelial lesion or malignancy, $p H P V$ HPV physician sampling, $s H P V$ HPV self-sampling

cases was $100 \%$, and HPV-16/-18 showed a higher CIN2+ rate than HPV others for self- and physician sampling. HPV$16 /-18$ with $57.1 \%$ (8/14) for CIN2+ physician sampling and $61.5 \%$ (8/13) for CIN2+ self-sampling as compared with HPV others at $15.8 \%(6 / 38)$ and $15.4 \%$ (6/39) for CIN2+, respectively.

\section{Discussion}

We investigated whether HPV self-sampling with an Evalyn brush and Cobas 4800 demonstrated HPV-type agreement with physician sampling and high detection sensitivity for CIN2+ in a Japanese referral population. The HPV-type concordance rate between sampling methods was $88 \%$ ( $\kappa: 0.76$ ), with HPV-16/-18 showing a higher match rate and $\kappa$ than HPV others. The detection sensitivity for CIN2+ by self-sampling was $100 \%$. The results showed that HPV self-sampling using an Evalyn brush and a Cobas 4800 PCR-based method might be feasible for cervical cancer screening in Japan.

In HPV self-sampling, participants collect the samples, making sample validity dependent on the patient. Therefore, it is important to compare HPV test results with those obtained from physician-collected samples considered the gold standard. The results of the present study showed a complete agreement rate for HPV typing of $88 \%$ ( $\kappa: 0.76$ ), consistent with a meta-analysis using a referral and screening population, regardless of detection methods and devices (87\%; $\kappa: 0.66)$ [9] and a Japanese referral population (84\%) [22]. Another study using the Evalyn brush and Cobas 4800 for ASCUS+ patients showed an agreement rate of $89.2 \%(\kappa$ : 0.70 ) [20], whereas a study using these devices to evaluate patients with premalignant lesions, cervical carcinoma, and carcinoma suspicion reported an agreement rate of $91 \%$ ( $\kappa$ : 0.64) [19]. In the present study, NILM/HPV-positive cases differed from these studies but showed a similar agreement rate. HPV-16/-18 is associated with malignant tumors [4, 23]. Therefore, it is important that self-sampling HPV-16/18 results are consistent with those of physician sampling. We found that HPV-16/-18 results showed higher agreement between sampling methods than HPV others, which was consistent with previous reports using the Evalyn brush and Cobas 4800 [20]. These findings demonstrated for the first time that HPV self-sampling using the Evalyn brush and Cobas 4800 agreed with physician sampling for HPV typing in the Japanese population.

Cases showing different results between sampling methods complicate the introduction of HPV self-sampling in screening. Most discrepant HPV tests involved NILM, and our results showed for the first time that discrepant cases using the Evalyn brush and Cobas 4800 in a Japanese population did not involve dysplasia. Inconsistencies in these cases associated with HPV testing between self- and physician sampling might be explained by self-sampling detecting HPV only in the vagina with no infected hrHPV in the cervix [10]. A previous study using Cobas 4800 showed that a high load of non-HPV16/HPV18 associated with the presence of dysplasia in physician-collected hrHPV. In cases without dysplasia, viral load was lower [24]; therefore, A lower load of hrHPV infection in cervix shed cells was impossible for their detection by self-sampling.

The sensitivity of CIN2+ detection by self-sampling was $100 \%$ and the same as physician sampling. A previous study evaluating sampling using the brush and PCR-based hrHPV testing by women referred for colposcopy due to
Table 5 CIN2+ detection sensitivity of HPV selfsampling, physician sampling, and cytology

\begin{tabular}{lcllr}
\hline & Sensitivity (\%) & Specificity (\%) & PPV (\%) & NPV (\%) \\
\hline sHPV & $100(66.1-100)$ & $58.1(47.0-68.7)$ & $26.5(14.9-41.1)$ & $100(89.6-100)$ \\
pHPV & $100(66.1-100)$ & $57.0(45.8-67.6)$ & $26.0(14.6-40.3)$ & $100(89.4-100)$ \\
ASCUS+ & $100(66.1-100)$ & $84.5(75.0-91.5)$ & $50.0(29.9-70.1)$ & $100(92.5-100)$ \\
LSIL+ & $92.3(64.0-99.8)$ & $89.3(80.6-95.0)$ & $57.1(34.0-78.2)$ & $98.7(92.9-100)$ \\
\hline
\end{tabular}

$A S C U S+$ atypical squamous cells of undetermined significance or worse, $H P V$ human papillomavirus, $L S I L+$ low-grade squamous intraepithelial lesion or worse, $N P V$ negative predictive value, $p H P V$ HPV physician sampling, $P P V$ positive predictive value, $s H P V$ HPV self-sampling 
abnormal cervical smear and/or post-coital bleeding with normal cytology showed a CIN2+-detection sensitivity of 93\% for self-sampling and $91 \%$ for physician sampling [25]. Another study evaluating the same methods in women that were previously hrHPV+ showed a CIN2+-detection sensitivity of $100 \%$ for both self- and physician sampling [26]. The results of the present study were consistent with these findings. Additionally, a study reported a lower CIN2+detection sensitivity with the Evalyn brush and HC methods for self-sampling relative to physician sampling in a Japanese referral population [27]. In the present study, the Evalyn brush and PCR-based method for self-sampling showed the same CIN2-detection sensitivity as physician sampling, supporting the superiority of PCR-based HPV testing for self-sampling in a Japanese population.

Previous studies indicated that CIN2+-detection sensitivity by self-sampling exceeded that of cytology in a referral population [15]; however, the present study revealed selfsampling as showing similar CIN2+-detection sensitivity to cytology, possibly due to the small number of CIN2+ cases in our study. Moreover, we found that both self- and physician sampling showed a lower CIN2+-detection specificity than cytology, reflecting either the possible presence of hrHPV infections not yet progressing to CIN2 or detection of vaginal hrHPV infection [10].

Here, samples collected with the Evalyn brush remained dried until transfer to a ThinPrep vial. Our result showed that the number of days from self-sampling to HPV testing did not influence discrepancies in HPV testing. The Evalyn brush is reportedly stable for the measurement of HPV DNA PCR results for up to 32 weeks [28], suggesting that discordant cases are independent of preservation methods.

Previous studies of HPV self-sampling targeted possible applications for cervical cancer screening; however, the high NPV value associated with HPV self-sampling for CIN2+ indicated that self-sampling might be useful for following up potential high-risk groups previously identified as HPV+. A previous study following HPV+ women reported an NPV for HSIL or worse was 98.8\% (95\% CI 91.6-99.8\%) in selfsampling and support self-sampling for exclusion of the disease during follow-up of HPV-positive women [29]. In the present study, the population had a high hrHPV+ rate and included follow-up patients that were previously HPV+. Using pathologic examination as the standard for NPV in our study revealed self-sampling as having a $100 \%$ NPV for CIN2+ detection, suggesting that a population at high risk for HPV might not require or delay regular visits upon acquisition of an HPV- result from self-sampling.

This study has some limitations. We examined a small number of referral cases, and sampling was not performed in a screening setting; however, to ensure the feasibility of HPV self-sampling using the Evalyn brush and Cobas 4800, it was necessary to evaluate match rates and CIN2+-detection sensitivity in a Japanese referral population. Therefore, studies in a Japanese screening population are needed. Additionally, HPV self-sampling was performed in a hospital and not at home, which would be the case in a screening setting. HPV-infection status can be affected not only by recent sexual acquisition or re-infection but also from recurrent detection of a controlled or latent infection [30]. Therefore, we attempted to determine whether physician and selfsampling were equally accurate when performed within the same timeframe. Two previous studies evaluating the Evalyn brush and Cobas 4800 involved home-based self-sampling, with their HPV concordance and CIN2+-detection rate consistent with those of our study involving hospital-based selfsampling $[18,20]$. Similar results might be obtained from home-based self-sampling in Japanese populations.

In conclusion, HPV self-sampling using an Evalyn brush and Cobas 4800 showed good agreement with physician sampling in a Japanese population. Moreover, the sensitivity of CIN2+ detection by self-sampling was as high as that for physician sampling using Cobas 4800 in a Japanese population. These results suggest that HPV self-sampling using the Evalyn brush and Cobas 4800 PCR-based testing methods might be efficacious for cervical cancer screening in Japan.

Acknowledgements We thank Professor Yasuo Ohashi and Mari Ito for helpful advice regarding the statistical methods. We also thank Hiroshi Konishi and Tadao Kakizoe in Japan Cancer Society for excellent advice for the organization.

Funding Research funding was received from Japan Cancer Society (public interest foundation).

\section{Compliance with ethical standards}

Conflict of interest The authors report no conflict of interest.

Open Access This article is licensed under a Creative Commons Attribution 4.0 International License, which permits use, sharing, adaptation, distribution and reproduction in any medium or format, as long as you give appropriate credit to the original author(s) and the source, provide a link to the Creative Commons licence, and indicate if changes were made. The images or other third party material in this article are included in the article's Creative Commons licence, unless indicated otherwise in a credit line to the material. If material is not included in the article's Creative Commons licence and your intended use is not permitted by statutory regulation or exceeds the permitted use, you will need to obtain permission directly from the copyright holder. To view a copy of this licence, visit http://creativecommons.org/licenses/by/4.0/.

\section{References}

1. Hanley SJ, Fujita H, Yokoyama S et al (2016) HPV self-sampling in Japanese women: a feasibility study in a population with limited experience of tampon use. J Med Screen 23:164-170. https://doi. org/10.1177/0969141315625702 
2. Motoki Y, Mizushima S, Taguri M et al (2015) Increasing trends in cervical cancer mortality among young Japanese women below the age of 50 years: an analysis using the Kanagawa populationbased Cancer Registry, 1975-2012. Cancer Epidemiol 39:700706. https://doi.org/10.1016/j.canep.2015.08.001

3. Spence AR, Goggin P, Franco EL (2007) Process of care failures in invasive cervical cancer: systematic review and meta-analysis. Prev Med 45:93-106

4. Bosch FX, Lorincz A, Muñoz N et al (2002) The causal relation between human papillomavirus and cervical cancer. J Clin Pathol 55:244-265

5. Wright TC Jr, Schiffman M, Solomon D et al (2004) Interim guidance for the use of human papillomavirus DNA testing as an adjunct to cervical cytology for screening. Obstet Gynecol 103:304-309

6. Ogilvie GS, van Niekerk D, Krajden M et al (2018) Effect of screening with primary cervical HPV testing vs cytology testing on high-grade cervical intraepithelial neoplasia at 48 months: the HPV FOCAL randomized clinical trial. JAMA 320:43-52. https ://doi.org/10.1001/jama.2018.7464

7. Lew JB, Simms KT, Smith MA et al (2017) Primary HPV testing versus cytology-based cervical screening in women in Australia vaccinated for HPV and unvaccinated: effectiveness and economic assessment for the National Cervical Screening Program. Lancet Public Health 2:e96-e107. https://doi.org/10.1016/S2468 -2667(17)30007-5

8. Maver PJ, Poljak M (2019) Primary HPV-based cervical cancer screening in Europe: implementation status, challenges, and future plans. Clin Microbiol Infect. https://doi.org/10.1016/j. cmi.2019.09.006

9. Petignat P, Faltin DL, Bruchim I et al (2007) Are self-collected samples comparable to physician-collected cervical specimens for human papillomavirus DNA testing? A systematic review and meta-analysis. Gynecol Oncol 105:530-535

10. Snijders PJ, Verhoef VM, Arbyn M et al (2013) High-risk HPV testing on self-sampled versus clinician-collected specimens: a review on the clinical accuracy and impact on population attendance in cervical cancer screening. Int J Cancer 132:2223-2236. https://doi.org/10.1002/ijc. 27790

11. Nelson EJ, Maynard BR, Loux T et al (2017) The acceptability of self-sampled screening for HPV DNA: a systematic review and meta-analysis. Sex Transm Infect 93:56-61. https://doi. org/10.1136/sextrans-2016-052609

12. Racey CS, Withrow DR, Gesink D (2013) Self-collected HPV testing improves participation in cervical cancer screening: a systematic review and meta-analysis. Can J Public Health 104:e159-e166

13. Verdoodt F, Jentschke M, Hillemanns P et al (2015) Reaching women who do not participate in the regular cervical cancer screening programme by offering self-sampling kits: a systematic review and meta-analysis of randomised trials. Eur J Cancer 51:2375-2385. https://doi.org/10.1016/j.ejca.2015.07.006

14. Polman NJ, Snijders PJF, Kenter GG et al (2019) HPV-based cervical screening: rationale, expectations and future perspectives of the new Dutch screening programme. Prev Med 119:108-117. https://doi.org/10.1016/j.ypmed.2018.12.021

15. Arbyn M, Verdoodt F, Snijders PJ et al (2014) Accuracy of human papillomavirus testing on self-collected versus clinician-collected samples: a meta-analysis. Lancet Oncol 15:172-183

16. van Baars R, Bosgraaf RP, ter Harmsel BW et al (2012) Dry storage and transport of a cervicovaginal self-sample by use of the Evalyn Brush, providing reliable human papillomavirus detection combined with comfort for women. J Clin Microbiol 50:39373943. https://doi.org/10.1128/JCM.01506-12

17. Polman NJ, Ebisch RMF, Heideman DAM et al (2019) Performance of human papillomavirus testing on self-collected versus clinician-collected samples for the detection of cervical intraepithelial neoplasia of grade 2 or worse: a randomised, paired screenpositive, non-inferiority trial. Lancet Oncol 20:229-238. https:// doi.org/10.1016/S1470-2045(18)30763-0

18. Wright TC, Stoler MH, Behrens CM et al (2015) Primary cervical cancer screening with human papillomavirus: end of study results from the ATHENA study using HPV as the first-line screening test. Gynecol Oncol 136:189-197. https://doi.org/10.1016/j.ygyno .2014.11.076

19. Leinonen MK, Schee K, Jonassen CM et al (2018) Safety and acceptability of human papillomavirus testing of self-collected specimens: a methodologic study of the impact of collection devices and HPV assays on sensitivity for cervical cancer and high-grade lesions. J Clin Virol 99-100:22-30. https://doi. org/10.1016/j.jcv.2017.12.008

20. Tranberg M, Jensen JS, Bech BH et al (2018) Good concordance of HPV detection between cervico-vaginal self-samples and general practitioner-collected samples using the Cobas $4800 \mathrm{HPV}$ DNA test. BMC Infect Dis 18:348. https://doi.org/10.1186/s1287 9-018-3254-y

21. Kurokawa T, Onuma T, Shinagawa A et al (2018) The ideal strategy for cervical cancer screening in Japan: result from the Fukui Cervical Cancer Screening Study. Cytopathology 29:361-367. https://doi.org/10.1111/cyt.12576

22. Yoshida T, Sano T, Takada N et al (2011) Comparison of selfcollected and clinician-collected materials for cervical cytology and human papillomavirus genotyping: analysis by linear array assay. Acta Cytol 55:106-112. https://doi.org/10.1159/000320924

23. Arbyn M, Ronco G, Anttila A et al (2012) Evidence regarding human papillomavirus testing in secondary prevention of cervical cancer. Vaccine 30:F88-F99. https://doi.org/10.1016/j.vacci ne.2012.06.095

24. Álvarez-Argüelles $\mathrm{ME}$, de Oña-Navarro $\mathrm{M}$, Rojo-Alba $\mathrm{S}$ et al (2015) Quantification of human papilloma virus (HPV) DNA using the Cobas 4800 system in women with and without pathological alterations attributable to the virus. J Virol Methods 222:95-102. https://doi.org/10.1016/j.jviromet.2015.05.016

25. Dijkstra MG, Heideman DA, van Kemenade FJ et al (2012) Brushbased self-sampling in combination with GP5+/6+-PCR-based hrHPV testing: high concordance with physician-taken cervical scrapes for HPV genotyping and detection of high-grade CIN. J Clin Virol 54:147-151. https://doi.org/10.1016/j.jcv.2012.02.022

26. Gustavsson I, Sanner K, Lindell M et al (2011) Type-specific detection of high-risk human papillomavirus (HPV) in self-sampled cervicovaginal cells applied to FTA elute cartridge. J Clin Virol 51:255-258. https://doi.org/10.1016/j.jcv.2011.05.006

27. Aiko KY, Yoko M, Saito OM et al (2017) Accuracy of self-collected human papillomavirus samples from Japanese women with abnormal cervical cytology. J Obstet Gynaecol Res 43:710-717. https://doi.org/10.1111/jog.13258

28. Ejegod DM, Pedersen H, Alzua GP et al (2018) Time and temperature dependent analytical stability of dry-collected Evalyn HPV self-sampling brush for cervical cancer screening. Papillomavirus Res 5:192-200. https://doi.org/10.1016/j.pvr.2018.04.005

29. Viviano M, Tran PL, Kenfack BJ et al (2018) Self- versus physician-collected samples for the follow-up of human papillomavirus-positive women in sub-Saharan Africa. Int J Womens Health 10:187-194. https://doi.org/10.2147/IJWH.S154212

30. Gravitt PE, Winer RL (2017) Natural history of HPV infection across the lifespan: role of viral latency. Viruses 9:e267. https:// doi.org/10.3390/v9100267

Publisher's Note Springer Nature remains neutral with regard to jurisdictional claims in published maps and institutional affiliations. 